\title{
The INSIDE Project: Innovative Solutions for In-Beam Dosimetry in Hadrontherapy
}

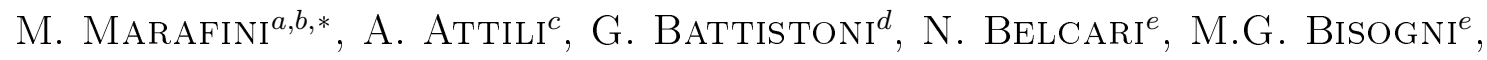

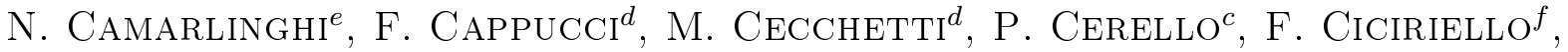

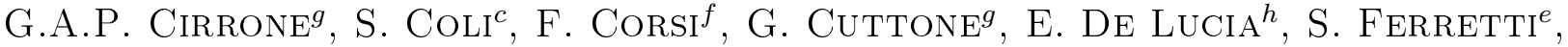

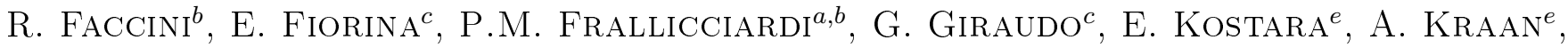

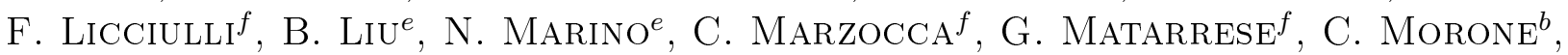
M. Morrocchi ${ }^{e}$, S. Muraro ${ }^{d}$, V. Patera ${ }^{b, i}$, F. Pennazio $^{c}$, C. Peroni $^{c}$, L. Piersanti $^{b, i}$,

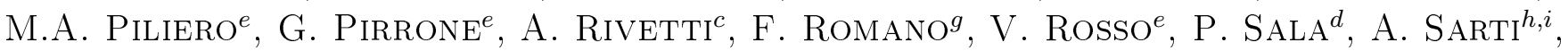

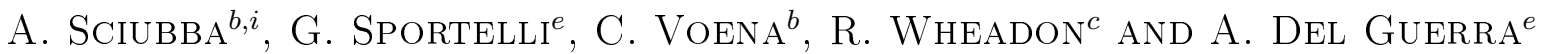

${ }^{a}$ Museo Storico della Fisica e Centro Studi e Ricerche "E. Fermi", Roma, Italy

${ }^{b-f}$ INFN Sezione di: ${ }^{b}$ Roma, Roma; ${ }^{c}$ Torino, Torino; ${ }^{d}$ Milano, Milano; ${ }^{e}$ Pisa, Pisa; ${ }^{f}$ Bari, Bari; Italy

${ }^{g}$ Laboratori Nazionali del Sud dell'INFN, Catania, Italy

${ }^{h}$ Laboratori Nazionali di Frascati dell'INFN, Frascati, Italy

${ }^{i}$ Dipartimento di Scienze di Base e Applicate per Ingegneria, Sapienza Università di Roma, Roma, Italy

The INSIDE project addresses the online dose monitoring in particle therapy issue. The proposed detectors exploit the secondary neutral and charged particles emitted during the treatment by the irradiated volumes. The new detection techniques allow to return and online a measurement of the released dose and the Bragg peak position.

DOI: $10.12693 /$ APhysPolA.127.1465

PACS: 87.53.Bn, 87.53.Jw, 87.53.Kn, 87.55.N-, 87.55.ne, 87.57.uq

\section{Particle therapy}

Particle therapy (PT) is an emerging technique $[1,2]$ alternative to conventional radiotherapy (RT), which makes use of charged particles, usually protons and carbon ions, to treat different kinds of static tumors. The most attracting feature of charged particles with respect to X-rays exploited in RT treatments is their dose profile, showing the highest released dose at the end of the track, in a region called Bragg peak (BP). The dose released in the entrance region is reduced with respect to RT, resulting in a better sparing of healthy tissue. Because the particle range is a function of the beam energy, using beams of different energies it is possible to accurately conform the dose to the tumor shape (spread out Bragg peak). Moreover, stopping carbon ions LETS have higher relative biological effectiveness (RBE) values with respect to X-rays, with corresponding higher efficiency in killing radio-resistant tumors.

\subsection{Monitoring with PET and charged particles}

Due to the high precision of PT, treatments are extremely sensitive to anatomical changes and patient mis-

\footnotetext{
* corresponding author; e-mail: michela.marafini@roma1.infn. it
}

positioning, with related risk of under-dosage of the cancer region or over-dosage of the healthy tissues. To overcome these issues, a dose monitor is eagerly needed. Unfortunately, unlike X-rays in conventional RT, the hadron beam is completely absorbed into the body, which means no possibility of using the fraction of beam escaping from the patient. A promising way to overcome this limitation is to detect secondary particles produced by the beam interaction into the body, namely charged particles, due to projectile and target fragmentation, prompt photons from nucleus de-excitation and pairs of back-toback photons produced by the annihilation of positrons coming from $\beta^{+}$emitters generated in the fragmentations. For all these different radiation types emitted it has been shown that the peak of the dose released can be correlated with the emission pattern of the flux of these secondary particles $[3,4]$.

\section{The INSIDE project}

The INSIDE (Innovative Solutions for In-Beam Dosimetry in hadrontherapy) MIUR project is born from the collaboration of a number of Italian Universities and INFN to build a multimodal in-beam dose monitoring system able to detect at the same time, back-to-back gammas from $\beta^{+}$annihilation and charged secondary particles with kinetic energy higher than $30 \mathrm{MeV}$ (prompt photons with energies higher than $1 \mathrm{MeV}$ can be exploited as well). The monitor will be made up of 2 planar of 
$10 \times 20 \mathrm{~cm}^{2}$ PET heads for back-to-back gammas detection and of a $20 \times 20 \mathrm{~cm}^{2}$ dual-mode dose profiler made of 3 sub-detectors: a tracker, an absorber and a calorimeter.

\subsection{PET system}

The goal of the PET-heads is to reconstruct the map of the $\beta^{+}$activity in-beam and inter-spill. The full in-beam
PET system must be able to sustain annihilation, prompt photon and neutron rates during the beam irradiation.

The dose distribution for both carbon ion and proton beam can be compared and univocally related to the $\beta^{+}$activity produced by the beam in the target. In Fig. 1 the supposition of the distributions is shown.
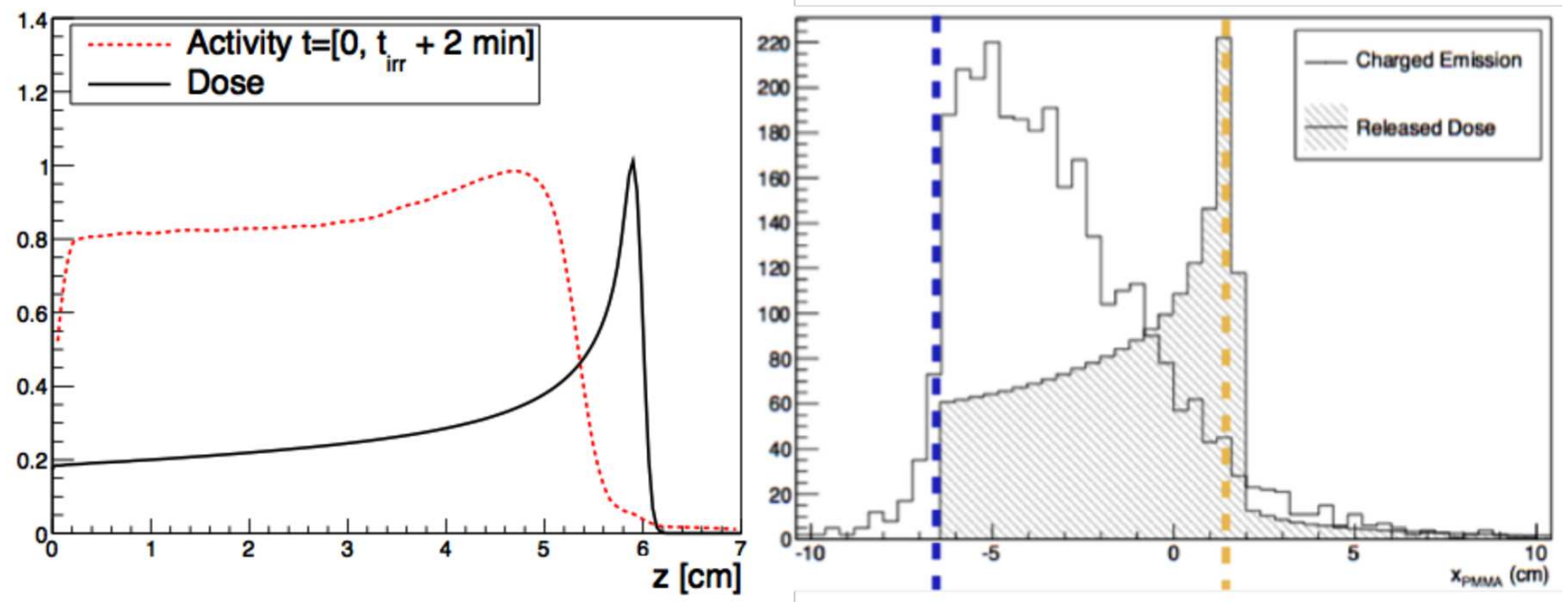

Fig. 1. Left: FLUKA MC simulation comparison between dose distribution (black solid line) superimposed to the longitudinal profile (red dotted line) of $\beta^{+}$activity for proton $95 \mathrm{MeV}$ beam on PMMA. Right: Comparison between the FLUKA simulated dose distribution (hatched) superimposed to the longitudinal profile (solid line) of charged secondary particles. In blue it is highlighted the position of PMMA entrance face of the beam, while in yellow it is indicated the BP abscissa position [4].

The two PET detectors are identical and are planar panels of $10 \times 20 \mathrm{~cm}^{2}$ each. They are made of $2 \times 4$ detection modules. Each module is composed of a pixelated LYSO matrix $16 \times 16$ pixels of $3 \times 3 \mathrm{~mm}^{2}$ crystals (pitch $3.1 \mathrm{~mm}$ ). The total sensitive area of a module will be $5 \times 5 \mathrm{~cm}^{2}$. The LYSO matrix readout will be performed by an array of SiPM $(16 \times 16$ pixels $)$ coupled one-to-one.

The resolution of the two PET heads system in the $\beta+$ activity reconstruction map is expected to be between 1 and $2 \mathrm{~mm}$ (FWHM) in beam direction.

\subsection{Dose profiler}

The aim of the profiler is to track and retrieve energy information of both of charged particles and of prompt gammas.

The BP position can be linked to the charged particles emission point. This allows to use the charged secondary particles information and to reconstruct the BP position during the treatment. Figure 1 (right) shows the dose delivered in superposition with charged particles emission point distribution. More details can be found in [3, 4].

The tracker will be made of $6 X Y$ planes of 384 $\left(0.5 \times 0.5 \mathrm{~mm}^{2}\right) \mathrm{BCF}-12$ plastic scintillating fibers, read out by S12571-050 Hamamatsu $1 \mathrm{~mm}^{2}$ SiPM read by
32 channel custom ASIC BASIC32-ADC. Behind the tracker, there will be positioned a $2.4 \mathrm{~mm}$ EJ-200 plastic scintillator slab to absorb the Compton electrons and to measure their energy. Backside of the absorber, the calorimeter will be made of $4 \times 4$ matrices of scintillating pixelated LYSO crystals. Each matrix is a $16 \times 16$ array of $2 \mathrm{~cm}$ thick LYSO read by a multi-anode H8500 PMT. An accurate MC simulation has optimized all the geometrical and physical parameters, exploiting out measurements as the starting point for the project design. The flux of secondary charged particles emitted from the target for a therapeutical treatments can be extrapolated combining the simulation informations with the measurements performed in last few years. From our calculations we expect to reach with the DP a final resolution of 1$2 \mathrm{~mm}$ in the emission point reconstruction.

\section{3. $C N A O$ setup}

The positioning of the different detectors in the treatment room will be performed with a cart in order to hold up the detectors at the optimized position and distance from the patient reducing any possible interference with the therapy procedures. In Fig. 2 we show the design of the cart and the different elements. 


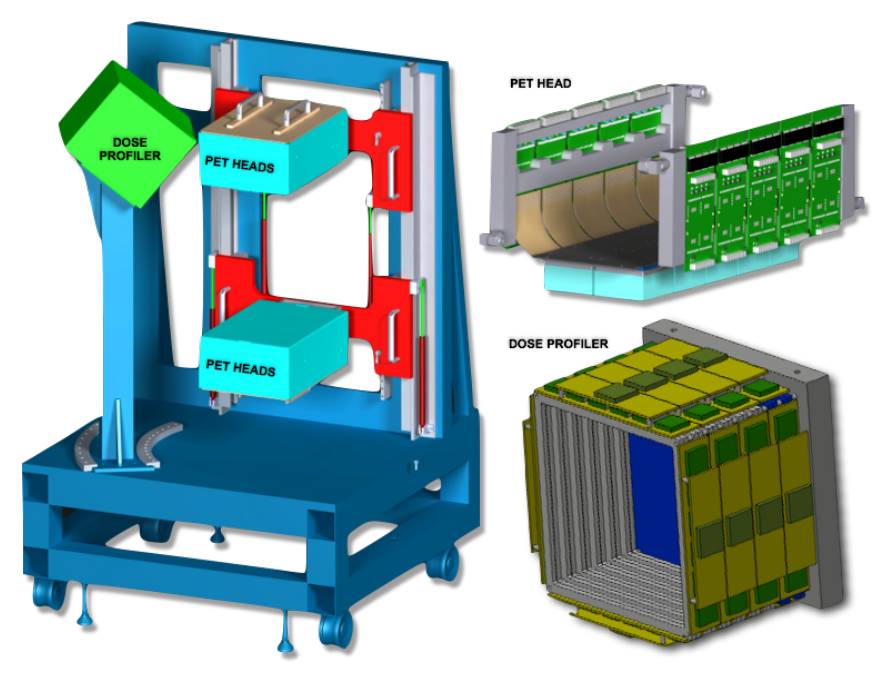

Fig. 2. The cart design for the CNAO treatment room: in light blue the two PET-heads with their movements (red), in green the Dose Profiler box (60 degrees from the beam line) and in blue the mechanical structure. On the right the prototype of a PET head and the mechanical structure of the DP.

\section{Acknowledgments}

This work has been partially supported by the European Commission under Contract FP7-PEOPLE-2012317446 .

\section{References}

[1] M. Durante, J.S. Loeffler, Nat. Rev. Clin. Oncol. 7, 37 (2010).

[2] U. Amaldi, G. Kraft, Rep. Prog. Phys. 68, 1861 (2005).

[3] C. Agodi, G. Battistoni, F. Bellini, G.A.P Cirrone, F. Collamati, G. Cuttone, E. De Lucia, M. De Napoli, A. Di Domenico, R. Faccini, F. Ferroni, S. Fiore, P. Gauzzi, E. Iarocci, M. Marafini, I. Mattei, S. Muraro, A. Paoloni, V. Patera, L. Piersanti, F. Romano, A. Sarti, A. Sciubba, E. Vitale C. Voena, Phys. Med. Biol. 57, 5667 (2012).

[4] L. Piersanti, F. Bellini, F. Bini, F. Collamati, E. De Lucia, M. Durante, R. Faccini, F. Ferroni, S. Fiore, E. Iarocci, C. La Tessa, M. Marafini, I. Mattei, V. Patera, P.G. Ortega, A. Sarti, C. Schuy, A. Sciubba, M. Vanstalle, C. Voena, Phys. Med. Biol. 59, 1857 (2013). 